\title{
Hidden symmetries in 5D supergravities and black rings
}

\author{
Dmitry V. Gal'tsov* \\ Moscow State University \\ E-mail: galtsov@phys.msu.ru
}

\section{Nikolai G. Scherbluk}

Moscow State University

E-mail: shcherbluck@mail.ru

\begin{abstract}
We construct generating technique for 5D minimal and $U(1)^{3}$ supergravities based on hidden symmetries arising in dimensional reduction to three dimensions. In the three-vector case the symmetry is $S O(4,4)$, and the minimal case corresponds to contraction of this group to $G_{2(2)}$. The matrix representation is presented applicable to both cases and the generating transformations preserving an asymptotic structure are listed. Our transformations contain enough free parameters to construct the general charged black ring in $U(1)^{3}$ theory starting with known solutions. To avoid a complicated inverse dualisation in the component form we introduce the matrix-valued dualisation which opens the way to derive new solutions purely algebraically from the coset representation of the seed.
\end{abstract}

Black Holes in General Relativity and String Theory

August 24-30 2008

Veli Losinj,Croatia

${ }^{*}$ Speaker. 


\section{Introduction}

Recent interest to black rings [1] stimulated search of new generating techniques for fivedimensional Einstein equations both vacuum and coupled to vector and/or scalar fields. An efficient tool to proliferate exact solutions to D-dimensional Einstein equations depending on three coordinates consists in dimensional reduction based on the assumption of existence of $D-3$ commuting Killing symmetries (toroidal reduction). Starting with D-dimensional Einstein equations coupled to scalar and vector fields one is able to derive a three-dimensional gravity coupled sigma-model in which the target space variables incorporate the initial scalars, vectors and moduli of the toroidal reduction. For a particular class of theories the target space turns out to be a coset space $G / H$ where $G$ is some semi-simple group known as the hidden symmetry group (for a recent review see [2]). This symmetry can be used to generate new solution form known ones with the same threedimensional metric. The sigma-model representation may also serve a basis of further reduction to two dimensions [3] (looking for solutions depending only on two variables), where more powerful methods can be developed such as inverse scattering technique [4]. Recently such an approach to vacuum five-dimensional relativity has undergone an impressive development [5] and resulted in construction of rather sophisticated ring configurations [6]. For charged rings no such technique was available so far, though generation via some restricted transformation involving vector fields were used [7]. Our matrix formulation opens a way to develop such methods in the general case of minimal and $U(1)^{3} 5 \mathrm{D}$ supergravities describing charged configurations.

Sigma-model generating technique for minimal five-dimensional supergravity was developed in $[8,9]$, for an earlier discussion of hidden symmetries in this theory see [10]. The hidden symmetry is this case is the non-compact version $G_{2(2)}$ of the lowest exceptional group $G_{2}$. To formulate the solution generating technique one has to use some matrix representation of the coset. Representing the seed solution in the matrix terms and acting by symmetry transformations one can extract the sigma-model variables for new solutions. In [8,9] an explicit $7 \times 7$ representation of the coset $G_{2(2)} / S L(2, R)^{2}$ was constructed using the representation of $G_{2}$ found by Gunyadin and Gursey [11]. The generalization to the case of five-dimensional supergravity with three $U(1)$ vector and two scalar fields was given in [12]. Apart from being more general, this theory is interesting by the fact that the corresponding hidden symmetry is given by a familiar group $S O(4,4)$. Actually, one of the ways to construct the matrix representation of $G_{2(2)}$ consists in using $S O(4,4)$ as a starting point [11] and imposing suitable constraints. The matrix representation of the relevant three-dimensional coset $S O(4,4) / S O(4) \times S O(4)$ is given in terms of the $8 \times 8$ matrices which are split into the $4 \times 4$ blocks. By freezing the scalar moduli and identifying the vector fields one reduces this theory to minimal $5 \mathrm{D}$ supergravity thus providing an alternative formulation of the technique of [8] in terms of the $8 \times 8$ matrices.

Generation of the new solution appeals to transformation of the target space variables by the hidden symmetry group. This part of the procedure is purely algebraic. Another part consists in solving the differential dualisation equations relating the target space variable to the metric and vector fields $[8,12]$. These equations have to be solved twice: first for the seed solution to obtain its description in terms of the coset matrix, and then for the transformed solution in order to extract the metric and the matter fields from the transformed coset matrix. Solving these equations may present technical difficulties on the second step if the generating transformations are complicated 
enough. To remedy this problem, we propose to pass to dualized variables in the matrix form. Such a possibility is suggested by the fact that the three-dimensional dual to the sigma-model matrixvalued current one-form is closed by virtue of the equations of motion. Then locally it is exact, and this provides the matrix-valued one-form whose exterior derivative is dual to the the initial matrix current. This dual matrix transforms under the global action of the hidden symmetry by some related transformation, and thus it can be found algebraically from the corresponding seed matrix. From this can read out the metric and matter fields of the transformed solution algebraically avoiding the inverse dualisation problem.

Our primary motivation to develop the generating techniques for 5D supergravity was lack of the general black ring solution possessing the electric charges (one in the minimal case and three in the $U(1)^{3}$ case), the magnetic charges, the mass and two independent rotation parameters [1]. In [8] an attempt was made to construct a charged black ring starting with the neutral solution with two rotation parameters found by Pomeranski and Senkov [13]. But the resulting solution was plagued with a conical singularity. To be able to derive a regular solution, one has to start with a non-regular seed solution with an extra free parameter, which can be fixed after the transformation. In principle, from the counting of free parameters in the transformations preserving asymptotic behavior of black rings, one finds that the general black ring can be generated indeed starting from some known solutions. But so far all attempts to find such a solution in a concise form were unsuccessful.

In this paper we illustrate the application of our technique generating new Kaluza-Klein squashed black holes. These black holes look as five-dimensional near the event horizon exhibiting the $S^{3}$ strucure, but asymptotically $S^{3}$ collapses to a twisted bundle of $S^{1}$ over $S^{2}$ with a constant radius of $S^{1}$ and growing radius of $S^{2}$. Thus at infinity they become four-dimensional objects with a compactified fifth dimension. One such solution to five-dimensional Einstein-Maxwell system was proposed by Ishihara and Matsuno [14] (non-rotating). Its physical parameters and thermodynamical properties were investigated in $[15,16]$. A certain class (but not all) of squashed black holes can be obtained by the so-called squashing transformation. This procedure was applied to asymptotically flat $[14,17,18]$ and non-asymptotically flat solutions such as Kerr-Gödel black holes [19, 20, 21]. In an attempt to enlarge the class of solutions, more recently Tomizawa, Yasui and Morisawa [22] applied $G_{2(2)}$ transformations of [8] to construct a generalization of the charged Rasheed black hole [23] obtaining a new solution with four independent parameters: mass, angular momentum, Kaluza-Klein parameter $\beta$ (in the notation of [23]) and an electric charge. Here we will derive a more general five-parametric solution adding as an independent parameter the quantity $\alpha$ of [23], which corresponds to an electric charge in the four-dimensional interpretation of the Rasheed solution.

\section{General setting}

The $U(1)^{3} 5 \mathrm{D}$ supergravity may be regarded as a truncated toroidal compactification of the 11D supergravity:

$$
I_{11}=\frac{1}{16 \pi G_{11}} \int\left(R_{11} \star_{11} 1-\frac{1}{2} F_{[4]} \wedge \star_{11} F_{[4]}-\frac{1}{6} F_{[4]} \wedge F_{[4]} \wedge A_{[3]}\right)
$$


where $F_{[4]}=d A_{[3]}$. Assuming an ansatz for the metric

$$
d s_{11}^{2}=d s_{5}^{2}+X^{1}\left(d z_{1}^{2}+d z_{2}^{2}\right)+X^{2}\left(d z_{3}^{2}+d z_{4}^{2}\right)+X^{3}\left(d z_{5}^{2}+d z_{6}^{2}\right),
$$

and the form field

$$
A_{[3]}=A^{1} \wedge d z_{1} \wedge d z_{2}+A^{2} \wedge d z_{3} \wedge d z_{4}+A^{3} \wedge d z_{5} \wedge d z_{6}
$$

where all functions are independent of $z$, we obtain the the bosonic sector of 5D supergravity coupled to three scalar moduli $X^{I}(I=1,2,3)$, satisfying the constraint $X^{1} X^{2} X^{3}=1$, and to three vector fields $A^{I}$ :

$$
\begin{aligned}
I_{5} & =\frac{1}{16 \pi G_{5}} \int\left(R_{5} \star_{5} 1-\frac{1}{2} G_{I J} d X^{I} \wedge \star_{5} d X^{J}-\frac{1}{2} G_{I J} F^{I} \wedge \star_{5} F^{J}-\frac{1}{6} \delta_{I J K} F^{I} \wedge F^{J} \wedge A^{K}\right), \\
G_{I J} & =\operatorname{diag}\left(\left(X^{1}\right)^{-2},\left(X^{2}\right)^{-2},\left(X^{3}\right)^{-2}\right), \quad F^{I}=d A^{I}, \quad I, J, K=1,2,3 .
\end{aligned}
$$

Here the Chern-Simons coefficients $\delta_{I J K}=1$ for the indices $I, J, K$ being a permutation of $1,2,3$, and zero otherwise. Contraction of the above theory to minimal $5 D$ supergravity is effected via an identification of the vector fields:

$$
A^{1}=A^{2}=A^{3}=\frac{1}{\sqrt{3}} A,
$$

and freezing out the moduli: $X^{1}=X^{2}=X^{3}=1$. This leads to the Lagrangian

$$
\mathscr{L}_{5}=R_{5} \star_{5} \mathbf{1}-\frac{1}{2} F \wedge \star_{5} F-\frac{1}{3 \sqrt{3}} F \wedge F \wedge A .
$$

It is worth noting that the 5D Einstein-Maxwell theory without the Chern-Simons term does not lead to the three-dimensional sigma model with a semi-simple hidden symmetry group, so in this case the solution generating technique can be formulated only for the static truncation of the theory. This explains why the charged rotating black hole solutions are not known analytically.

\subsection{Four-dimensional view}

Consider reduction of the $\mathrm{D}=5$ action (2.3) to four dimensions. We assume that the $5 \mathrm{D}$ spacetime has the structure $\mathscr{M}_{5}=\mathscr{M}_{4} \times S^{1}$, where $S^{1}$ is a circle, and is parameterized by the coordinates $\left\{x^{\mu}, z\right\}, \mu=1, \ldots, 4$ with $z$ relating to the circle. Following to the standard procedure we decompose the $5 \mathrm{D}$ metric as

$$
d s_{5}^{2}=e^{\frac{\phi}{\sqrt{3}}} d s_{4}^{2}+e^{-\frac{2 \phi}{\sqrt{3}}}(d z+a)^{2}
$$

where the is $d s_{4}^{2}=g_{\mu v}(x) d x^{\mu} d x^{v}$, the Kaluza-Klein one-form is $a=a_{\mu} d x^{\mu}$ and $\phi$ is the dilaton. In a similar way the $5 \mathrm{D}$ vector fields $A^{I}\left(x^{\mu}, z\right)$ are decomposed as

$$
A^{I}\left(x^{\mu}, z\right)=A^{I}\left(x^{\mu}\right)+u^{I} d z
$$

where $u^{I}$ are the axions. All the above fields do not depend on $z$. Inserting these decompositions into the $5 \mathrm{D}$ action we get the $4 \mathrm{D}$ lagrangian

$$
\begin{aligned}
\mathscr{L}_{4} & \left.=R_{4} \star 1-\frac{1}{2} \star d \phi \wedge d \phi-\frac{1}{2} G_{I J} \star d X^{I} \wedge d X^{J}-\frac{1}{2} e^{\frac{2 \phi}{\sqrt{3}}} G_{I J} \star d u^{I} \wedge d u^{J}-\frac{1}{2} e^{-\sqrt{3} \phi} \star \mathscr{F} \wedge \mathscr{F} 2.6\right) \\
& -\frac{1}{2} e^{-\frac{\phi}{\sqrt{3}}} G_{I J} \star F^{I} \wedge F^{J}-\frac{1}{2} \delta_{I J K} d A^{I} \wedge d A^{J} u^{K},
\end{aligned}
$$


where $\mathscr{F}=d a$ and $F^{I}=d A^{I}-d u^{I} \wedge a$ are the field strength two-forms. Our purpose is to rewrite this lagrangian in the form exhibiting the S-duality symmetry. First of all we consider the scalar part of (2.6) written in the following form

$$
e_{4}^{-1} \mathscr{L}_{\text {scal }}=\frac{1}{2}\left((\partial \phi)^{2}+G_{I J} \partial X^{I} \partial X^{J}+e^{\frac{2 \phi}{\sqrt{3}}} G_{I J} \partial u^{I} \partial u^{J}\right)=\hat{\mathscr{G}}_{A B}(\hat{\Phi}) \partial \hat{\Phi}^{A} \partial \hat{\Phi}^{B}, \quad A, B=1, \ldots, 6
$$

where $\partial \equiv \partial / \partial x^{\mu}, e_{4}$ is the Hodge dual to unity: $e_{4} \equiv \star 1=\sqrt{-g} d^{4} x$ and all index operations refer to the metric $g_{\mu \nu}$. The potentials $\hat{\Phi}^{A}$ combine the six variables $\left\{X^{1}, X^{2}, \phi, u^{I}\right\}$ and realize the map $\hat{\Phi}^{A}: x^{\mu} \in \mathscr{M}_{4} \rightarrow \hat{\Phi}^{A}\left(x^{\mu}\right) \in \mathscr{M}_{\text {scal }}$ between the 4D Minkowskian space-time and the target space with the metric $\hat{\mathscr{G}}_{A B}(\hat{\Phi})$. Replacing the dilaton $\phi$ and the moduli $X^{I}$ by the new variables $\alpha^{I}$ : $\alpha^{I}=\phi / \sqrt{3}-\ln X^{I}$ enable us to simplify $\mathscr{L}_{\text {scal }}$ as follows

$$
e_{4}^{-1} \mathscr{L}_{\text {scal }}=\frac{1}{2} \sum_{I}\left(\left(\partial \alpha^{I}\right)^{2}+e^{2 \alpha^{I}}\left(\partial u^{I}\right)^{2}\right) .
$$

The structure of the scalar manifold $\mathscr{M}_{\text {scal }}$ becomes more transparent in terms of three complex potentials $z^{I}=u^{I}+i e^{-\alpha^{I}}$ :

$$
e_{4}^{-1} \mathscr{L}_{\text {scal }}=\frac{1}{2} \sum\left|\partial z^{I}\right|^{2} /\left(\operatorname{Im} z^{I}\right)^{2}
$$

The lagrangian $\mathscr{L}=\frac{1}{2}|\partial z|^{2} /(\operatorname{Im} z)^{2}$ invariant under the group $S L(2, \mathbb{R})$ and the corresponding target space metric is the Kähler space $S L(2, \mathbb{R}) / S O(2)$. So in our case the isometry group of $\mathscr{M}_{\text {scal }}$ is $\hat{G}=(S L(2, \mathbb{R}))^{3}$ and the corresponding target space is $\hat{G} / \hat{H}=\mathscr{M}_{\text {scal }}=(S L(2, \mathbb{R}) / S O(2))^{3}$ with the metric

$$
\hat{\mathscr{G}}_{A B}(\hat{\Phi}) d \hat{\Phi}^{A} d \hat{\Phi}^{B}=\frac{1}{2}\left((d \phi)^{2}+G_{I J} d X^{I} d X^{J}+e^{\frac{2 \phi}{\sqrt{3}}} G_{I J} d u^{I} d u^{J}\right)=\frac{1}{2} \sum\left|d z^{I}\right|^{2} /\left(\operatorname{Im} z^{I}\right)^{2} .
$$

As the second step, we reformulate the vector part of the lagrangian (2.6) according with the structure of the bosonic lagrangian of $N=2$ supergravity coupled to vector multiplets (for a review see the Ref.[24]). We express it in terms of the field two-forms $\widetilde{F}^{I}$ and $\mathscr{F}$ obeying to the Bianchi identities $d \widetilde{F}^{I}=0$ and $d \mathscr{F}=0$ respectively. To extract the two-forms $\widetilde{F}^{I}$ one has to combine the exterior derivative $d\left(u^{I} a\right)$ in $F^{I}=d A^{I}-d u^{I} \wedge a$. As result we have $F^{I}=\widetilde{F}^{I}+u^{I} \mathscr{F}$, where $\widetilde{F}^{I}=d \widetilde{A} \equiv d\left(A^{I}-u^{I} a\right)$. Inserting the two-forms $F^{I}$ and $d A^{I}$ expressed via $\widetilde{F}$ and $\mathscr{F}$ into (2.6) and integrating by parts the terms $\delta_{I J K} \widetilde{F}^{I} \wedge d u^{J} u^{K} \wedge a$ and $\delta_{I J K} d u^{I} u^{J} u^{K} \wedge a \wedge \mathscr{F}$ we will obtain for the vector part of the $4 \mathrm{D}$ lagrangian

$$
\begin{aligned}
\mathscr{L}_{\text {vect }} & =\frac{1}{2}\left[e^{-\sqrt{3} \phi} \star \mathscr{F} \wedge \mathscr{F}+e^{-\frac{\phi}{\sqrt{3}}} G_{I J}\left(\star \widetilde{F}^{I} \wedge \widetilde{F}^{J}+2 \star \mathscr{F} u^{[I} \wedge \widetilde{F}^{J]}+u^{I} u^{J} \star \mathscr{F} \wedge \mathscr{F}\right)\right. \\
& \left.+\delta_{I J K}\left(\widetilde{F}^{I} \wedge \widetilde{F}^{J} u^{K}+\widetilde{F}^{I} u^{J} u^{K} \wedge \mathscr{F}+\frac{1}{3} u^{I} u^{J} u^{K} \mathscr{F} \wedge \mathscr{F}\right)\right] .
\end{aligned}
$$

Denote the field strength and its Hodge dual as $\mathscr{F}=\frac{1}{2} \mathscr{F}_{\mu \nu} d x^{\mu} \wedge d x^{v}$ and $\star \mathscr{F}_{\mu \nu}=\frac{1}{2} \mathscr{F} \alpha \beta \varepsilon_{\alpha \beta \mu \nu}$, where $\varepsilon_{\alpha \beta \mu \nu}$ is the totally antisymmetric Levi-Civita tensor with $(-g)^{1 / 2}$. Assuming that $d x^{\mu} \wedge$ $d x^{v} \wedge d x^{\alpha} \wedge d x^{\beta}=-\varepsilon^{\mu v \alpha \beta} e_{4}$, we find

$$
\star \mathscr{F} \wedge \mathscr{F}=\frac{1}{2} \mathscr{F}_{\mu v} \mathscr{F}^{\mu v} e_{4}=\frac{1}{2} \mathscr{F}^{2} e_{4}, \quad \mathscr{F} \wedge \mathscr{F}=-\frac{1}{2} \mathscr{F} \mu v \star \mathscr{F}^{\mu v} e_{4}=-\frac{1}{2} \mathscr{F} \star \mathscr{F} e_{4} .
$$


Note that in the 4D Lorentzian signature space the double Hodge dual is $\star \star=-1$. We then combine the field tensors $\widetilde{F}_{\mu v}^{I}$ and $\mathscr{F}_{\mu \nu}$ into the 4-column $\mathscr{B}_{\mu v}=\left(\begin{array}{c}\widetilde{F}_{\mu v}^{I} \\ \mathscr{F}_{\mu v}\end{array}\right)$ and rewrite (2.7) in the matrix form adopted in $[25,26]$ :

$$
e_{4}^{-1} \mathscr{L}_{\text {vect }}=\frac{1}{4} \mathscr{B}_{\alpha \beta}^{T}\left(\hat{\mu} \mathscr{B}^{\alpha \beta}-\frac{1}{\sqrt{2}} \hat{v} \star \mathscr{B}^{\alpha \beta}\right)
$$

where the symmetric $4 \times 4$ matrices $\hat{\mu}$ and $\hat{v}$ are given by

$$
\hat{\mu}=\left(\begin{array}{cc}
e^{-\frac{\phi}{\sqrt{3}}} G_{I J} & e^{-\frac{\phi}{\sqrt{3}}} G_{I J} u^{J} \\
e^{-\frac{\phi}{\sqrt{3}}} G_{I J} u^{J} & G_{I J} u^{I} u^{J}+e^{-\sqrt{3} \phi}
\end{array}\right), \quad \hat{v}=\sqrt{2}\left(\begin{array}{cc}
\delta_{I J K} u^{K} & \frac{1}{2} \delta_{I J K} u^{J} u^{K} \\
\frac{1}{2} \delta_{I J K} u^{J} u^{K} & 2 u^{1} u^{2} u^{3}
\end{array}\right)
$$

This lagrangian yields the field equations for $\mathscr{B}_{\alpha \beta}^{T}: \nabla_{\alpha}\left(\hat{\mu} \mathscr{B}^{\alpha \beta}-\frac{1}{\sqrt{2}} \hat{v} \star \mathscr{B}^{\alpha \beta}\right)=0$. Introducing the dual field strength $\mathscr{H}_{\alpha \beta}$ as $\star \mathscr{H}^{\alpha \beta}=\hat{\mu} \mathscr{B}^{\alpha \beta}-\frac{1}{\sqrt{2}} \hat{v} \star \mathscr{B}^{\alpha \beta}$ we see that the above equations are the Bianchi identities for $\mathscr{H}_{\alpha \beta}$. Therefore the lagrangian $\mathscr{L}_{\text {vect }}$ takes the form manifestly S-duality symmetric:

$$
e_{4}^{-1} \mathscr{L}_{\text {vect }}=\frac{1}{4} \mathscr{B}_{\alpha \beta}^{T} \star \mathscr{H}^{\alpha \beta}=\frac{1}{8} \mathfrak{J}^{T} \Sigma_{1} \star \mathfrak{I}, \quad \mathfrak{I}=\left(\begin{array}{c}
\mathscr{B}_{\alpha \beta} \\
\mathscr{H}_{\alpha \beta}
\end{array}\right), \quad \Sigma_{1}=\left(\begin{array}{ll}
0 & 1 \\
1 & 0
\end{array}\right) .
$$

It can be checked that relation between $\mathfrak{I}$ and $\star \mathfrak{I}$ is given by

$$
\mathfrak{I}=\Omega \hat{P} \star \mathfrak{I}
$$

where $\Omega=\left(\begin{array}{cc}0 & 1 \\ -1 & 0\end{array}\right)$ is the $8 \times 8$ symplectic metric and $\hat{P}$ is the $8 \times 8$ matrix depending on the potentials of the scalar manifolds $\mathscr{M}_{\text {scal }}$

$$
\hat{P}=\left(\begin{array}{cc}
\hat{\mu}+\hat{v} \hat{\mu}^{-1} \hat{v} & \hat{v} \hat{\mu}^{-1} \\
\hat{\mu}^{-1} \hat{v} & \hat{\mu}^{-1}
\end{array}\right)
$$

The matrix $\hat{P}$ provides the representation $\gamma$ of the coset element $\pi\left(\hat{\Phi}^{A}\right)$, namely $\gamma: \pi \in \mathscr{M}_{\text {scal }} \rightarrow$ $\gamma(\pi)=\hat{P}$. We then have

$$
\hat{\mathscr{G}}_{A B} d \hat{\Phi}^{A} d \hat{\Phi}^{B}=-\frac{1}{16} \operatorname{Tr}\left(d \hat{P} d \hat{P}^{-1}\right)=-\frac{1}{8} \operatorname{Tr}\left(d \mu d \mu^{-1}-d v \mu^{-1} d v \mu^{-1}\right)
$$

Consider diffeomorphism $\hat{\Phi}^{A} \rightarrow \hat{\Phi}^{A \prime}$, which leave invariant the target space metric. It corresponds to the action of some element $\hat{g}$ belonging to the isometry group of the target space $\hat{g} \in \hat{G}$. In terms of the matrix representation $\gamma$ this means that the coset matrix $\hat{R} \equiv \Sigma_{1} \hat{P}$ transforms as $\hat{R} \rightarrow \hat{R}^{\prime}=\gamma(\hat{g}) \hat{R} \gamma\left(\hat{g}^{-1}\right)$. Inserting the expression $\star \mathfrak{I}=-\Omega \hat{P} \mathfrak{I}$ into the $\mathscr{L}_{\text {tens }}$ and keeping in mind that $\Sigma_{1} \Omega=-\Omega \Sigma_{1}$ we will obtain for the tensor part of the lagrangian:

$$
e_{4}^{-1} \mathscr{L}_{\text {vect }}=\frac{1}{8} \mathfrak{I}^{T} \Omega \hat{R} \mathfrak{I}
$$


If we now demand this lagrangian to be invariant under the action of $\gamma(\hat{g})$, we get the restrictions for the element $\widetilde{g} \in \hat{G}$ acting on the column as $\mathfrak{I} \rightarrow \gamma(\widetilde{g}) \mathfrak{I}$. Performing the transformation we have

$$
\mathscr{L}_{\text {vect }} \rightarrow \mathscr{L}_{\text {vect }}^{\prime}=\frac{1}{8} \mathfrak{J}^{T} \gamma(\widetilde{g})^{T} \Omega \gamma(\hat{g}) \hat{R} \gamma\left(\hat{g}^{-1}\right) \gamma(\widetilde{g}) \mathfrak{I} .
$$

Thus the conditions for $\gamma(\widetilde{g})$ are $\gamma(\widetilde{g})=\gamma(\hat{g})$ and $\gamma(\hat{g})^{T} \Omega \gamma(\hat{g})=\Omega$. This relation means that there is the symplectic embedding of the isometry group into the symplectic group $\hat{G} \rightarrow S p(8, \mathbb{R})$ [27]. In other words, $\gamma(\hat{g})$ provides the symplectic representation of $\hat{g}$ which rotates the fields $\mathfrak{I}$. Note the full $4 \mathrm{D}$ lagrangian can be written in the following form

$$
e_{4}^{-1} \mathscr{L}_{4}=R_{4}+\frac{1}{16} \operatorname{Tr}\left(\partial \hat{R} \partial \hat{R}^{-1}\right)-\frac{1}{8} \mathfrak{J}^{T} \Omega \hat{R} \mathfrak{I}
$$

Thus the S-duality group for the four-dimensional reduction of the $U(1)^{3}$ supergravity is $\operatorname{SL}(2, R)^{3}$, reducing to $S L(2, R)$ in the minimal case.

\section{3D sigma-model}

Consider now further reduction to three dimensions. It is convenient to restart from 11D supergravity. An overall assumption for the 11D manifold will be $\mathscr{M}_{11}=T^{6} \times \Sigma \times \mathscr{M}_{3}$ where $\Sigma$ is $T^{2}$ if both these Killing vectors are asymptotically space-like, or $T^{1} \times \mathbb{R}$ if one of them is asymptotically time-like. The full set of $11 \mathrm{D}$ coordinates $x^{N}, N=1, \ldots, 11$ is thus split into $z^{a} \in$ $T^{6}, a=1, \ldots, 6, x^{i} \in \mathscr{M}_{3}, i=1, \ldots, 3$ and $z^{p} \in \Sigma, p=7,8$. The decomposition of the 5D metric is given by

$$
d s_{5}^{2}=\lambda_{p q}\left(d z^{p}+a^{p}\right)\left(d z^{q}+a^{q}\right)-\kappa \tau^{-1} h_{i j} d x^{i} d x^{j},
$$

where all metric functions are independent on $z^{a}$ and $z^{p}$. The 5D metric components are parameterized by the KK one-forms $a^{p}=a_{i}^{p} d x^{i}$, the three-dimensional metric $h_{i j}$ of $\mathscr{M}_{3}$ and the scalars $\varphi_{1}, \varphi_{2}, \chi$, which are arranged in the following $2 \times 2$ matrix

$$
\lambda=\mathrm{e}^{-\frac{2}{\sqrt{3}} \varphi_{1}}\left(\begin{array}{cc}
1 & \chi \\
\chi & \chi^{2}+\kappa \mathrm{e}^{\sqrt{3} \varphi_{1}-\varphi_{2}}
\end{array}\right), \quad \operatorname{det} \lambda \equiv-\tau=\kappa \mathrm{e}^{-\frac{1}{\sqrt{3}} \varphi_{1}-\varphi_{2}}
$$

where $\kappa= \pm$ is responsible for the signature: $\kappa=1$ for space-like $z^{8}$, and $\kappa=-1$ for time-like $z^{8}$. The ansatz (2.2) leads to the five-dimensional action (2.3). The 5D $U(1)$ gauge fields $A^{I}$ reduce to the 3D one-forms $A^{I}\left(x^{i}\right)$ and the six axions collectively denoted as the 2D-covariant doublet $\psi_{p}^{I}=\left(u^{I}, v^{I}\right)$ with the index $p$ relative to the metric $\lambda_{p q}$

$$
A^{I}\left(x^{i}, z^{7}, z^{8}\right)=A^{I}\left(x^{i}\right)+\psi_{p}^{I} d z^{p}=A^{I}\left(x^{i}\right)+u^{I} d z^{7}+v^{I} d z^{8} .
$$

To obtain the three-dimensional sigma-model one has to dualize the electro-magnetic (EM) one-forms $A^{I}$ and the KK one-forms $a^{p}$ to scalars, which will be denoted as $\mu_{I}$ and $\omega_{p}$. The dualisation equations read:

$$
\begin{gathered}
\tau \lambda_{p q} d a^{q}=\star V_{p}, \\
d A^{I}=d \psi_{q}^{I} \wedge a^{q}+\tau^{-1} G^{I J} \star G_{J},
\end{gathered}
$$


where the one-forms $G_{I}$ and $V_{p}$ are given by

$$
\begin{aligned}
& G_{I}=d \mu_{I}+\frac{1}{2} \delta_{I J K} d \psi_{p}^{J} \psi_{q}^{K} \varepsilon^{p q} \\
& V_{p}=d \omega_{p}-\psi_{p}^{I}\left(d \mu_{I}+\frac{1}{6} \delta_{I J K} d \psi_{q}^{J} \psi_{r}^{K} \varepsilon^{q r}\right) .
\end{aligned}
$$

In the component form the Eqs.(3.2) read: ${ }^{1}$

$$
\begin{aligned}
& \lambda_{p q} \partial^{[i} a^{j] q}=\frac{1}{2 \tau \sqrt{h}} \varepsilon^{i j k}\left[\partial_{k} \omega_{p}-\psi_{p}^{I}\left(\partial_{k} \mu_{I}+\frac{1}{6} \delta_{I J K} \partial_{k} \psi_{r}^{J} \psi_{t}^{K} \varepsilon^{r t}\right)\right], \\
& \partial^{\left[{ }^{i} A^{j] I}\right.}=a^{q[j} \partial^{i]} \psi_{q}^{I}+\frac{1}{2 \tau \sqrt{h}} \varepsilon^{i j k} G^{I J}\left(\partial_{k} \mu_{J}+\frac{1}{2} \delta_{J K L} \partial_{r} \psi_{p}^{K} \psi_{q}^{L} \varepsilon^{p q}\right) .
\end{aligned}
$$

Substituting the metric $d s_{5}^{2}$ in the form (3.1) into the 5D action (2.3) and performing dualisation via Eqs.(3.2) one derive the 3D gravity coupled sigma-model:

$$
I_{3}=\frac{1}{16 \pi G_{3}} \int \sqrt{|h|}\left(R_{3}-\mathscr{G}_{A B} \frac{\partial \Phi^{A}}{\partial x^{i}} \frac{\partial \Phi^{B}}{\partial x^{j}} h^{i j}\right) d^{3} x
$$

where the Ricci scalar $R_{3}$ is build using the 3-dimensional metric $h_{i j}$. The set of potentials ${ }^{2} \Phi^{A}=$ $\left(\vec{\phi}, \psi^{I}, \mu_{I}, \chi, \omega_{p}\right), A, B=1, \ldots, 16$ realizes the harmonic map $\Phi^{A}: x^{i} \in \mathscr{M}_{3} \rightarrow \Phi^{A}\left(x^{i}\right) \in \mathscr{M}_{\text {scal }}$ between the $3 \mathrm{D}$ space-time $\mathscr{M}_{3}$ and the target space $\mathscr{M}_{\text {scal }}$ with the metric $\mathscr{G}_{A B}\left(\Phi^{C}\right)$. The target space line element $d l^{2}=\mathscr{G}_{A B} d \Phi^{A} d \Phi^{B}$ has the form

$$
\begin{aligned}
d l^{2} & =\frac{1}{2} G_{I J}\left(d X^{I} d X^{J}+d \psi^{I^{T}} \lambda^{-1} d \psi^{J}\right)-\frac{1}{2} \tau^{-1} G^{I J} G_{I} G_{J}+\frac{1}{4} \operatorname{Tr}\left(\lambda^{-1} d \lambda \lambda^{-1} d \lambda\right) \\
& +\frac{1}{4} \tau^{-2} d \tau^{2}-\frac{1}{2} \tau^{-1} V^{T} \lambda^{-1} V .
\end{aligned}
$$

It is invariant under the action of the 28-parametric isometry group $S O(4,4)$. The target space manifold $\mathscr{M}_{\text {scal }}$ is isomorphic to the coset $\mathscr{M}=S O(4,4) / H$, where the isotropy group $H$ is $S O(4) \times$ $S O(4)$ for $\kappa=1$ and $S O(2,2) \times S O(2,2)$ for $\kappa=-1$. That is there is an isomorphic map $\pi$ : $\Phi^{A} \rightarrow \pi\left(\Phi^{A}\right) \in \mathscr{M}$. Moreover if $g \in S O(4,4)$ is some constant element of the isometry group then the following transformations

$$
\pi \rightarrow \pi^{\prime}=g \circ \pi, \quad d s_{3}^{2} \rightarrow d s_{3}^{2}
$$

leave invariant the action (3.4).

As a convenient representative of the coset $\pi\left(\Phi^{A}\right) \in \mathscr{M}$ one can choose the matrix representation $\gamma: \pi \rightarrow \gamma(\pi) \equiv \mathscr{V}$, where $\mathscr{V}$ is the upper triangular matrix. We assume that $\mathscr{V}$ transforms

\footnotetext{
${ }^{1}$ the antisymmetrization is assumed with $1 / 2$.

${ }^{2}$ The set $\vec{\phi}=\left(\phi_{1}, \phi_{2}, \phi_{3}, \phi_{4}\right)$ comprises four scalars related to previously introduced $\varphi_{1}, \varphi_{2}, \chi$ and $X^{I}$ via

$$
\begin{aligned}
\phi_{1} & =\frac{1}{\sqrt{2}}\left(-\ln \left(X^{3}\right)+\frac{1}{\sqrt{3}} \varphi_{1}+\varphi_{2}\right), & \phi_{2} & =\frac{1}{\sqrt{2}}\left(\ln \left(X^{3}\right)-\frac{1}{\sqrt{3}} \varphi_{1}+\varphi_{2}\right), \\
\phi_{3} & =\frac{1}{\sqrt{2}}\left(\ln \left(X^{3}\right)+\frac{2}{\sqrt{3}} \varphi_{1}\right), & \phi_{4} & =\frac{1}{\sqrt{2}} \ln \frac{X^{1}}{X^{2}} .
\end{aligned}
$$
}


under the global action of the symmetry group $S O(4,4)$ by the right multiplication and under the local action of the isotropy group $H$ by the left multiplication: $\mathscr{V} \rightarrow \mathscr{V}^{\prime}=h(\Phi) \mathscr{V} g$, where $g$ and $h$ belong to the matrix representation $\gamma$ of $S O(4,4)$ and $H$ respectively. Given this representative, one can construct the $H$-invariant matrix (which we denote the same symbol $\mathscr{M}$ as the coset space)

$$
\mathscr{M}=\mathscr{V}^{T} K \mathscr{V}
$$

where $K$ is an involution matrix invariant under $H: h(\Phi)^{T} K h(\Phi)=K$, and dependent on the coset signature parameter $\kappa$. Then the transformation of the matrix $\mathscr{M}$ under $S O(4,4)$ will be

$$
\mathscr{M} \rightarrow \mathscr{M}^{\prime}=g^{T} \mathscr{M} g
$$

The target space metric (3.5) in terms of the matrix $\mathscr{M}$ will read

$$
d l^{2}=-\frac{1}{8} \operatorname{Tr}\left(d \mathscr{M} d \mathscr{M}^{-1}\right) .
$$

Choosing suitable $8 \times 8$ matrix representation $\gamma$ of the isometry group $S O(4,4)$ we construct (see [12] for details ) the matrix representation of the coset $\mathscr{M}$ in terms of the $4 \times 4$ block matrices $\mathscr{P}=\mathscr{P}^{T}$ and $\mathscr{Q}=-\mathscr{Q}^{\widehat{T} 3}$ as follows

$$
\mathscr{M}=\left(\begin{array}{cc}
\mathscr{P} & \mathscr{P} \mathscr{Q} \\
\mathscr{Q}^{T} \mathscr{P} & \widetilde{P}+\mathscr{Q}^{T} \mathscr{P} \mathscr{Q}
\end{array}\right),
$$

where the block matrices are given explicitly in the Appendix.

\subsection{Matrix dualisation}

As we have discussed, the dualisation equations (3.3) may present difficulties in applications of the solution generating technique. We can improve the situation performing dualisation in the matrix form. Introducing the matrix-valued current one-form $\mathscr{J}$

$$
\mathscr{J}=\mathscr{J}_{i} d x^{i}=\mathscr{M} d \mathscr{M}^{-1}
$$

we can rewrite the 3-dimensional sigma-model action (3.4) in the following form

$$
I_{3}=\frac{1}{16 \pi G_{3}} \int\left(R_{3} \star 1-\frac{1}{8} \operatorname{Tr}(\mathscr{J} \wedge \star \mathscr{J})\right) .
$$

In this expression the Hodge dual $\star$ is assumed with respect to the 3-dimensional metric $h_{i j}$. Variation of this action with respect to $\mathscr{J}$ shows that the two-form $\star \mathscr{J}$ is closed:

$$
d \star \mathscr{J}=0 .
$$

Variation with respect to the metric leads to three-dimensional Einstein equations:

$$
\left(R_{3}\right)_{i j}=\frac{1}{8} \operatorname{Tr}\left(\mathscr{J}_{i} \mathscr{J}_{j}\right)
$$

\footnotetext{
${ }^{3} \widehat{T}$ denotes transposition with respect to the minor diagonal
} 
The first equation (3.8) means that the matrix-valued two-forms $\star \mathscr{J}$ is locally exact, i.e., it can be presented as the exterior derivative of some matrix-valued one-form $\mathscr{N}$, that is

$$
\star \mathscr{J}=\mathscr{M} \star d \mathscr{M}^{-1}=d \mathscr{N} .
$$

The matrix $\mathscr{N}$ is defined up to adding an arbitrary matrix-valued closed one-form, which can be determined by choosing suitable asymptotic conditions. Now comparing the matrix dualisation equation (3.10) with the initial dualisation equations (3.3) we find the following purely algebraic relations between certain components of the matrix $(\mathscr{N})_{a b}, a, b=1, \ldots, 8$ are and the previous variables $a^{p}$ and $A^{I}$, namely

$$
\begin{aligned}
& a^{7}=(\mathscr{N})_{16}, \quad a^{8}=(\mathscr{N})_{17}, \\
& A^{1}=\psi_{p}^{1} a^{p}+(\mathscr{N})_{15}, \quad A^{2}=\psi_{p}^{2} a^{p}+(\mathscr{N})_{14}, \quad A^{3}=\psi_{p}^{3} a^{p}-(\mathscr{N})_{26} .
\end{aligned}
$$

Thus, if one manages to find the matrix $\mathscr{N}$, the metric and matter fields can be extracted algebraically.

For the following it is important that the definition (3.10) and the transformation law for the matrix $\mathscr{M}$ (3.6) under the global transformations $g \in S O(4,4)$ imply the following transformation of the matrix $\mathscr{N}$ :

$$
\mathscr{N} \rightarrow \mathscr{N}^{\prime}=g^{T} \mathscr{N}\left(g^{T}\right)^{-1} .
$$

Using (3.11 one can read off the metric components and potentials without explicitly solving the differential dualisation equations.

\section{Solution generating technique}

The sigma-model presented in the previous sections gives rise to generating technique which allows to construct new solutions from the known ones. Let the metric $h_{i j}$ and the set of potentials $\Phi^{A}$ combined in the coset matrix $\mathscr{M}$ correspond to the metric and the three-form of some 11D seed solution. One has to extract part of the target space potentials from the seed solution algebraically and solve the differential dualisation equations (3.2) to find the remaining potentials. Using the action of the target space isometries one can then construct a new solution of the sigma-model with the same three-metric $h_{i j}^{\prime}=h_{i j}$ and the coset matrix

$$
\mathscr{M}^{\prime}=g^{T} \mathscr{M} g \quad\left(\text { or } \mathscr{M}^{\prime}=g \mathscr{M}^{T}\right), \quad g \in S O(4,4) .
$$

Note that five target space variables $\phi_{1}, \phi_{2}, \phi_{3}, \phi_{4}, \chi$ enter the eleven-dimensional metric algebraically, via the moduli $X^{I}, \lambda_{p q}$ :

$$
d s_{11}^{2}=\sum_{I, a, a^{\prime}} X^{I}\left(\left(d z^{a}\right)^{2}+\left(d z^{a^{\prime}}\right)^{2}\right)+\lambda_{p q}\left(d z^{p}+a^{p}\right)\left(d z^{q}+a^{q}\right)+\tau^{-1} h_{i j} d x^{i} d x^{j}, a a^{\prime}=(12,34,56)
$$

while the KK vectors $a^{p}$ in the $T^{2}$ sector are related to the target space potentials $\omega_{p}$ via dualisation. Similarly, in the form-field sector,

$$
A_{[3]}=\left(A^{1}+\psi_{p}^{1} d z^{p}\right) \wedge d z^{1} \wedge d z^{2}+\left(A^{2}+\psi_{p}^{2} d z^{p}\right) \wedge d z^{3} \wedge d z^{4}+\left(A^{3}+\psi_{p}^{3} d z^{p}\right) \wedge d z^{5} \wedge d z^{6}
$$


the six quantities $\psi_{p}^{I}$ are the target space potentials, while the remaining one forms $A^{I}$ are related to the potentials $\mu_{I}$ via dualisation. So the set of transformed potentials $\lambda_{p q}^{\prime},\left(X^{I}\right)^{\prime}$ and $\left(\psi_{p}^{I}\right)^{\prime}$ can be explicitly extracted from the coset matrix $\mathscr{M}^{\prime}$. The remaining components of the transformed metric $\left(d s_{11}^{2}\right)^{\prime}$ and the 3 -form $\left(A_{[3]}\right)^{\prime}$ which are parametrized as the KK one-forms $\left(a^{p}\right)^{\prime}$ and the EM fields $\left(A^{I}\right)^{\prime}$ are determined by the dualisation equations (3.2). The inverse dualisation via the Eqs. (3.3) may be very difficult technically. Fortunately, this problem can be reduced to a purely algebraic one using the dualisation in the matrix form (3.10) as described in the previous section. Taking into account that the matrix $\mathscr{N}$ transforms as

$$
\mathscr{N}^{\prime}=g^{T} \mathscr{N}\left(g^{T}\right)^{-1} \quad\left(\text { or } \mathscr{N}^{\prime}=g \mathscr{N} g^{-1}\right), \quad g \in S O(4,4)
$$

and using the relations (3.11) one can easily obtain the desired quantities $\left(a^{p}\right)^{\prime}$ and $\left(A^{I}\right)^{\prime}$.

We will denote the 28 generators of the $s o(4,4)$ algebra as

$$
\mathscr{T}=\left(H_{1}, H_{2}, H_{3}, H_{4}, P^{ \pm I}, W_{ \pm I}, Z_{ \pm I}, \Omega^{ \pm p}, X^{ \pm}\right),
$$

with $I=1,2,3, p=7,8$. Their matrix representation can be found in the Appendix. The corresponding one-parametric transformations $g=\mathrm{e}^{\alpha \mathscr{T}}$, where $\alpha$ is a transformation parameter, give the set of the target space isometries.

\subsection{Asymptotic conditions}

An important question is how to identify the isometries we need to use in order to construct solutions with the desired properties. These are usually associated with asymptotic conditions. In this paper we consider asymptotic conditions corresponding to 5D Kaluza-Klein black holes with squashed horizons embedded into eleven dimensions which correspond to the following asymptotic manifold: $T^{6} \times \mathbb{R}^{1} \times S_{s q}$, where $S_{s q}$ is a squashed $S^{3}$. We will assume that target space potentials have the following general asymptotic behavior

$$
\lambda \sim\left(\begin{array}{cc}
1 & 0 \\
0 & -1
\end{array}\right)+\frac{\delta \lambda}{r}, \quad \omega_{7} \sim \frac{\delta \omega_{7}}{r}, \quad \omega_{8} \sim \frac{\delta \omega_{8}}{r^{2}}, \quad A_{[3]}=0,
$$

where $\delta \lambda, \delta \omega_{7}$ and $\delta \omega_{8}$ are constant. The asymptotic behavior with $\delta \lambda=\delta \omega_{7}=\delta \omega_{8}=0$ correspond to the trivial $S^{1}$ bundle over a $4 \mathrm{D}$ Minkowski space-time. The asymptotic coset matrix for this case is $\mathscr{M}_{a s}=K$ which is preserved under the isometries belonging to the isotropy group $H$ of the $S O(4,4)$ :

$$
P^{I}+P^{-I}, \quad Z_{I}+Z_{-I}, \quad W_{I}-W_{-I}, \quad X^{+}+X^{-}, \quad \Omega^{7}+\Omega^{-7}, \quad \Omega^{8}-\Omega^{-8} .
$$

For more general asymptotic behavior such as (4.1) one have use the above transformations with some constraints on the parameters.

To apply these isometries in the case of minimal 5D supergravity one needs to find the relevant embedding of the $G_{2(2)}$ subgroup into $S O(4,4)$. As was shown in [12], the following combinations of the $S O(4,4)$ generators realize the positive and negative root generators of $G_{2(2)}$ :

$$
P^{ \pm} \sim \sum P^{ \pm I}, \quad Z_{ \pm} \sim \sum Z_{ \pm I}, \quad W_{ \pm} \sim \sum W_{ \pm I}, \quad \Omega^{ \pm p}, \quad X^{ \pm} .
$$

Thus the isometries

$$
P^{+}+P^{-}, \quad Z_{+}+Z_{-}, \quad W_{+}-W_{-}, \quad X^{+}+X^{-}, \quad \Omega^{7}+\Omega^{-7}, \quad \Omega^{8}-\Omega^{-8}
$$

can be used to generate new KK solutions in the minimal 5D supergravity. 


\section{Five-parametric squashed black hole}

Our improved generating technique allows us to construct the charged Rasheed solution from the Kerr Black Hole. We define the coordinates $z^{7}=x^{5}, z^{8}=t$ and $x^{i}=(r, \theta, \phi)$. In this basis the Kerr solution of the mass $M_{K}$ and the angular momentum $J_{K}=a M_{K}$ smeared into the fifth dimension reads

$$
d s_{5}^{2}=\left(d x^{5}\right)^{2}-(1-Z)\left(d t+\frac{a Z \sin ^{2} \theta}{1-Z} d \phi\right)^{2}+\frac{\rho}{\Delta} d r^{2}+\rho d \theta^{2}+\frac{\Delta}{1-Z} \sin ^{2} \theta d \phi^{2},
$$

where

$$
\rho=r^{2}+a^{2} \cos ^{2} \theta, \quad \Delta=r^{2}-2 M_{K} r+a^{2}, \quad Z=\frac{2 M_{K} r}{\rho} .
$$

The corresponding target space variables are:

$$
\begin{aligned}
& \lambda_{p q}=\left(\begin{array}{cc}
1 & 0 \\
0 & Z-1
\end{array}\right), \quad \tau=1-Z, \\
& \omega_{7}=0, \quad \omega_{8}=\frac{2 M_{K} a \cos \theta}{\rho}, \quad\left(a_{\phi}^{7}=0, \quad a_{\phi}^{8}=\frac{a Z \sin ^{2} \theta}{1-Z}\right) .
\end{aligned}
$$

The above definitions of the target space potentials lead to the following blocks of the coset matrix $\mathscr{M}$

$$
\mathscr{Q}=\left(\begin{array}{llll}
0 & 0 & \frac{2 M_{K} a \cos \theta}{\rho} & 0 \\
0 & 0 & 0 & 0 \\
0 & 0 & 0 & 0 \\
0 & 0 & 0 & 0
\end{array}\right), \quad \mathscr{P}=\left(\begin{array}{cccc}
\frac{1}{Z-1} & 0 & 0 & 0 \\
0 & \frac{1}{Z-1} & 0 & 0 \\
0 & 0 & 1 & 0 \\
0 & 0 & 0 & 1
\end{array}\right) .
$$

One can easily obtain the dual matrix $\mathscr{N}$ solving the Eq.(3.10) :

$$
\mathscr{N}=\left(\begin{array}{cccccccc}
-\frac{2 M_{K} \Delta \cos \theta}{\rho(1-Z)} & 0 & 0 & 0 & 0 & 0 & \frac{Z a \sin ^{2} \theta}{1-Z} & 0 \\
0 & -\frac{2 M_{K} \Delta \cos \theta}{\rho(1-Z)} & 0 & 0 & 0 & 0 & 0 & -\frac{Z a \sin ^{2} \theta}{1-Z} \\
0 & 0 & 0 & 0 & 0 & 0 & 0 & 0 \\
0 & 0 & 0 & 0 & 0 & 0 & 0 & 0 \\
0 & 0 & 0 & 0 & 0 & 0 & 0 & 0 \\
0 & 0 & 0 & 0 & 0 & 0 & 0 & 0 \\
-\frac{Z a\left(r-2 M_{k}\right) \sin ^{2} \theta}{r(1-Z)} & 0 & 0 & 0 & 0 & 0 & \frac{2 M_{K} \Delta \cos \theta}{\rho(1-Z)} & 0 \\
0 & \frac{Z a\left(r-2 M_{k}\right) \sin ^{2} \theta}{r(1-Z)} & 0 & 0 & 0 & 0 & 0 & \frac{2 M_{K} \Delta \cos \theta}{\rho(1-Z)}
\end{array}\right) d \phi .
$$

To obtain the charged dyon solution from the Kerr one we apply to the seed coset matrices $\mathscr{M}$ and $\mathscr{N}$ the following sequence of global transformations

$$
g_{1}=\mathrm{e}^{\alpha\left(X^{+}+X^{-}\right)} \rightarrow g_{2}=\mathrm{e}^{\beta\left(\Omega^{7}+\Omega^{-7}\right)} \rightarrow g_{3}=\mathrm{e}^{\gamma\left(\Omega^{8}-\Omega^{-8}\right)} \rightarrow g_{4}=\mathrm{e}^{\delta \sum_{I}\left(Z_{I}+Z_{-I}\right)}
$$

with the constant parameters $\alpha, \beta, \gamma, \delta$. Here we assume that the matrices $\mathscr{M}$ and $\mathscr{N}$ are transformed under $g=g_{1} g_{2} g_{3} g_{4}$ as $\mathscr{M}^{\prime}=g \mathscr{M} g^{T}$ and $\mathscr{N}^{\prime}=g \mathscr{N} g^{-1}$ respectively. Then we demand that $g_{1} g_{2} g_{3}$ preserve the $O\left(\frac{1}{r}\right)$ asymptotic behavior of $a_{\phi}^{8}$ or, equivalently, the $O\left(\frac{1}{r^{2}}\right)$ asymptotic behavior of $\omega_{8}$. This give the same relation between three parameters $\alpha, \beta, \gamma$ as in [23]:

$$
\tan 2 \gamma=\tanh \alpha \sinh \beta
$$


This constraint ensures the asymptotic flatness and the absence of the NUT parameter in the fourdimensional solution. Then extracting the target space variables from $\mathscr{M}^{\prime}$ and $\mathscr{N}^{\prime}$, transformed KK one-forms $\left(a^{p}\right)^{\prime}$ and the five-dimensional one-form $A^{\prime}$ one can write the metric and the 3-form field of new solution:

$$
\begin{aligned}
d s_{11}^{2} & =\sum_{a, a^{\prime}}\left(\left(d z^{a}\right)^{2}+\left(d z^{a^{\prime}}\right)^{2}\right) \\
& +f\left(d t+\Omega^{\prime}\right)^{2}+\frac{1}{f D}\left(d x^{5}+W d \phi\right)^{2}-D\left(\frac{\rho}{\Delta} d r^{2}+\rho d \theta^{2}+\frac{\Delta}{1-Z} \sin ^{2} \theta d \phi^{2}\right), \\
A_{[3]}^{\prime} & =\sum_{a, a^{\prime}} \frac{c s}{D}\left\{(A+B) d t-(s C+c E) d x^{5}+[c(X B-W E)-s(W C+Y A)] d \phi\right\} \wedge d z^{a} \wedge d z^{a^{\prime}},
\end{aligned}
$$

with

$$
\begin{aligned}
& f=\frac{A B}{D^{2}}, \quad \Omega^{\prime}=\Omega_{5} d x^{5}+\Omega_{\phi} d \phi, \quad D=A c^{2}+B s^{2} \\
& \Omega_{5}=\frac{C}{A} s^{3}-\frac{E}{B} c^{3}, \quad \Omega_{\phi}=\frac{W C+Y A}{A} s^{3}+\frac{X B-W E}{B} c^{3} .
\end{aligned}
$$

The functions $A, B, C, E, X, Y, W$ is given by

$$
\begin{aligned}
& A=\frac{2 M_{K}^{2} c_{\beta}^{2}\left(c_{\alpha}-p\right)+2 M_{K}\left(r\left(p-c_{\alpha} c_{\beta}^{2}\right)-a s_{\alpha} s_{\beta} c_{\beta}^{2} \cos \theta\right)-p \rho}{p\left(\rho-2 M_{k} r\right)}, \\
& B=\frac{2 M_{K}^{2}\left(1+c_{\alpha} p\right)\left(p-c_{\alpha} c_{\beta}^{2}\right)+2 M_{K}\left(a s_{\alpha} s_{\beta}\left(1+c_{\alpha}^{2} c_{\beta}^{2}\right) \cos \theta-r\left(c_{\alpha} p^{2}-c_{\alpha} c_{\beta}^{2}+p\right)\right)-p \rho}{p\left(\rho-2 M_{k} r\right)} \\
& E=\frac{2 M_{K}\left(M_{K} s_{\alpha}\left(c_{\alpha} c_{\beta}^{2}-p\right)+r p s_{\alpha}-a c_{\alpha} s_{\beta} \cos \theta\right)}{\rho-2 M_{K} r}, \\
& C=-\frac{2 M_{K} c_{\beta}\left(M_{K} s_{\alpha} s_{\beta}+a p \cos \theta\right)}{\rho-2 M_{K} r}, \\
& W=\frac{2 M_{K} c_{\beta}\left\{M_{K}\left(a s_{\alpha} \sin ^{2} \theta\left(p-c_{\alpha} c_{\beta}^{2}\right)-2 s_{\beta} r \cos \theta\right)+s_{\beta}\left(r^{2}+a^{2}\right) \cos \theta-a s_{\alpha} p r \sin ^{2} \theta\right\}}{p\left(\rho-2 M_{K} r\right)} \\
& X=-\frac{2 M_{K} a c_{\beta} \sin ^{2} \theta\left(M_{K}\left(c_{\alpha}-p\right)-r c_{\alpha}\right)}{\rho-2 M_{K} r}, \\
& Y=-2 M_{K}\left\{M_{K}\left(2 s_{\alpha} p r \cos \theta-a s_{\beta} \sin ^{2} \theta\left(p c_{\alpha}+1\right)\right)-s_{\alpha} p \cos \theta\left(a^{2}+r^{2}\right)+a s_{\beta} r \sin ^{2} \theta\right\} \\
& \rho-2 M_{K} r
\end{aligned}
$$

where

$$
p=\sqrt{c_{\alpha}^{2}+s_{\alpha}^{2} s_{\beta}^{2}}, \quad c_{\star}=\cosh \star, s_{\star}=\sinh \star, \quad c=\cosh \delta, s=\sinh \delta .
$$

Our new solution contains five free parameters $M_{K}, a, \alpha, \beta, \delta$ and reduces to that of [22] if $\alpha=0$. 


\section{Asymptotic behavior}

The functions (5.1)-(5.7) have the following asymptotic behavior at spatial infinity

$$
\begin{aligned}
A & =-1-\frac{2 M_{K} c_{\beta}^{2} c_{\alpha}}{p} \frac{1}{r}+O\left(\frac{1}{r^{2}}\right) \\
B & =1+\frac{2 M_{K} c_{\alpha}\left(c_{\beta}^{2}-p^{2}\right)}{p} \frac{1}{r}+O\left(\frac{1}{r^{2}}\right) \\
C & =-2 M_{K} c_{\beta}\left(s_{\alpha} s_{\beta} M_{K}+p a \cos \theta\right) \frac{1}{r^{2}}+O\left(\frac{1}{r^{3}}\right) \\
E & =2 M_{K} s_{\alpha} p \frac{1}{r}+O\left(\frac{1}{r^{2}}\right) \\
X & =2 M_{K} a c_{\alpha} c_{\beta} \sin ^{2} \theta \frac{1}{r}+O\left(\frac{1}{r^{2}}\right) \\
Y & =2 M_{k} s_{\alpha} p \cos \theta-2 M_{K} a s_{\beta} \sin ^{2} \theta \frac{1}{r}+O\left(\frac{1}{r^{2}}\right) \\
W & =\frac{2 M_{K} s_{\beta} c_{\beta} \cos \theta}{p}-2 M_{K} a c_{\beta} s_{\alpha} \sin ^{2} \theta \frac{1}{r}+O\left(\frac{1}{r^{2}}\right)
\end{aligned}
$$

These decompositions lead to the asymptotical expression of the five-dimensional metric and the electro-magnetic one-form $A^{\prime}$ :

$$
\begin{aligned}
d s_{5}^{2} & =-d t^{2}+\left(d x^{5}+\frac{2 M_{K} s_{\beta} c_{\beta} \cos \theta}{p} d \phi\right)^{2}+d r^{2}+r^{2}\left(d \theta^{2}+\sin ^{2} \theta d \phi^{2}\right), \\
A_{[3]}^{\prime} & =\sum_{a, a^{\prime}} A^{\prime} \wedge d z^{a} \wedge d z^{a^{\prime}}, \\
A_{t}^{\prime} & =\frac{2 M_{K} c s p c_{\alpha}}{r}+O\left(\frac{1}{r^{2}}\right), \quad A_{x^{5}}^{\prime}=\frac{2 M_{K} c^{2} s p s_{\alpha}}{r}+O\left(\frac{1}{r^{2}}\right), \\
A_{\phi}^{\prime} & =2 M_{K} c s\left\{-s p s_{\alpha} \cos \theta+\frac{a \sin ^{2} \theta\left(s s_{\beta}-c c_{\alpha} c_{\beta}\right)+2 M_{K} s_{\alpha} \cos \theta\left(c c_{\beta} s_{\beta}+s^{3} p^{2} c_{\alpha}\right)}{r}\right\}+O\left(\frac{1}{r^{2}}\right),
\end{aligned}
$$

Then we define the Komar mass and angular momenta as

$$
\begin{aligned}
M & =\frac{1}{2 \pi^{2}} \int d \Sigma_{\alpha \beta} \xi_{(t)}^{\alpha ; \beta}, \\
J_{\phi} & =-\frac{1}{2 \pi^{2}} \int d \Sigma_{\alpha \beta} \xi_{(\phi)}^{\alpha ; \beta}, \\
J_{x^{5}} & =-\frac{1}{2 \pi^{2}} \int d \Sigma_{\alpha \beta} \xi_{\left(x^{5}\right)}^{\alpha ; \beta},
\end{aligned}
$$

where $\xi_{(t)}^{\alpha}, \xi_{(\phi)}^{\alpha}, \xi_{\left(x^{5}\right)}^{\alpha}$ are the Killing vector fields $\xi_{(t)}=\xi_{(t)}^{\alpha} \partial_{\alpha}=\partial_{t}, \xi_{(\phi)}=\partial_{\phi}, \xi_{\left(x^{5}\right)}=\partial_{x^{5}}$, which normalized as $\xi_{(t)}^{2}=-1, \xi_{(\phi)}^{2}=1, \xi_{\left(x^{5}\right)}^{2}=1$ at infinity. The integrals are taken over the squashed $S^{3}$ at spatial infinity $r \rightarrow \infty$ and the surface element is $d \Sigma_{t r}=r^{2} \sin (\theta) d \theta \wedge d \phi \wedge d x^{5}$. Here we assume that $x^{5} \in S^{1}$ has the periodicity $2 \pi R_{5}$. The computations of the Komar integrals with respect the 5-dimensional metric

$$
d s_{5}^{2}=f\left(d t+\Omega^{\prime}\right)^{2}+\frac{1}{f D}\left(d x^{5}+W d \phi\right)^{2}-D\left(\frac{\rho}{\Delta} d r^{2}+\rho d \theta^{2}+\frac{\Delta}{1-Z} \sin ^{2} \theta d \phi^{2}\right)
$$


give the following results:

$$
\begin{aligned}
M & =8 R_{5}\left(c^{2}+s^{2}\right) M_{K} p c_{\alpha} \\
J_{\phi} & =-\frac{4}{3} R_{5} M_{K} a\left(c_{\alpha} c_{\beta} c^{3}-s_{\beta} s^{3}\right), \\
J_{x^{5}} & =-4 R_{5} M_{K} p s_{\alpha} c^{3}
\end{aligned}
$$

The conserved electric charge $Q_{e}$ of the new solution is

$$
Q_{e}=-\frac{1}{4 \pi G_{5}} \int d \Sigma_{\alpha \beta}\left(F^{\prime} \alpha \beta+\frac{1}{\sqrt{3} \sqrt{-g}} \varepsilon^{\alpha \beta \gamma \delta \eta} A_{\gamma}^{\prime} F_{\delta \eta}^{\prime}\right)
$$

where $F^{\prime}=d A^{\prime}$. One finds that

$$
Q_{e}=\frac{8 \pi}{G_{5}} R_{5} M_{K} s c c_{\alpha} p
$$

\section{Conclusions}

We have presented a new formulation of solution generating technique for the $5 \mathrm{D}$ minimal and $U(1)^{3}$ supergravities based on the 3D sigma-model with the $S O(4,4)$ isometry group. Starting from any seed solution possessing two commuting Killing vector fields and using transformations of the target space isometry group one can construct new solutions with the same three-dimensional metric. The solution generation procedure consists in solving the dualisation equations for the seed solution to express it in the sigma-model variables, applying some $S O(4,4)$ transformations to get new sigma-model potential, and finally to pass back to the metric and field variable. Usually the last steps also involves solving the dualisation equations, but we suggest here the dualisation in the matrix form with an independent transformation of the dual variables. This allows to avoid solving differential equations for the backward dualisation, replacing this step by an algebraic procedure. As an application we have obtained the five-parametric Kaluza-Klein black hole of the minimal $5 \mathrm{D}$ supergravity. Our generating transformations generalize those of the vacuum $5 \mathrm{D}$ gravity to the presence of vector fields and open a way to develop the inverse scattering technique for this more general case.

\section{Acknowledgments}

The authors are grateful to Gerard Clément and Chiang-Mei-Chen for helpful discussions. The paper was supported by the RFBR grant 08-02-01398-a.

\section{A. $8 \times 8$ matrix representation}

We choose the following $8 \times 8$ matrix representation of the so(4,4) algebra

$$
E=\left(\begin{array}{cc}
A & B \\
C & -A^{\widehat{T}}
\end{array}\right),
$$


where $A, B, C$ are the $4 \times 4$ matrices, $A, B$ being antisymmetric, $B=-B^{T}, C=-C^{T}$, and the symbol $\widehat{T}$ in $A^{\widehat{T}}$ means transposition with respect to the minor diagonal. The diagonal matrices $\vec{H}$ are given by the following $A$-type matrices (with $B=0=C$ ):

$$
A_{H_{1}}=\left(\begin{array}{cccc}
\sqrt{2} & 0 & 0 & 0 \\
0 & 0 & 0 & 0 \\
0 & 0 & 0 & 0 \\
0 & 0 & 0 & 0
\end{array}\right), A_{H_{2}}=\left(\begin{array}{cccc}
0 & 0 & 0 & 0 \\
0 & \sqrt{2} & 0 & 0 \\
0 & 0 & 0 & 0 \\
0 & 0 & 0 & 0
\end{array}\right), A_{H_{3}}=\left(\begin{array}{cccc}
0 & 0 & 0 & 0 \\
0 & 0 & 0 & 0 \\
0 & 0 & \sqrt{2} & 0 \\
0 & 0 & 0 & 0
\end{array}\right), A_{H_{4}}=\left(\begin{array}{cccc}
0 & 0 & 0 & 0 \\
0 & 0 & 0 & 0 \\
0 & 0 & 0 & 0 \\
0 & 0 & 0 & \sqrt{2}
\end{array}\right) .
$$

Twelve generators corresponding to the positive roots are given by the upper-triangular matrices $E_{k}, k=1, \ldots, 12$,. From these the generators labeled by $k=2,4,6,7,9,12$ are of pure $A$-type (with $B=0=C$ ):

$$
\begin{aligned}
& A_{E_{2}}=\left(\begin{array}{llll}
0 & 0 & 0 & 1 \\
0 & 0 & 0 & 0 \\
0 & 0 & 0 & 0 \\
0 & 0 & 0 & 0
\end{array}\right), \quad A_{E_{4}}=\left(\begin{array}{cccc}
0 & 0 & 0 & 0 \\
0 & 0 & 0 & 0 \\
0 & 0 & 0 & -1 \\
0 & 0 & 0 & 0
\end{array}\right), \quad A_{E_{6}}=\left(\begin{array}{llll}
0 & 1 & 0 & 0 \\
0 & 0 & 0 & 0 \\
0 & 0 & 0 & 0 \\
0 & 0 & 0 & 0
\end{array}\right), \\
& A_{E_{7}}=\left(\begin{array}{cccc}
0 & 0 & 0 & 0 \\
0 & 0 & 0 & -1 \\
0 & 0 & 0 & 0 \\
0 & 0 & 0 & 0
\end{array}\right) \quad A_{E_{9}}=\left(\begin{array}{cccc}
0 & 0 & -1 & 0 \\
0 & 0 & 0 & 0 \\
0 & 0 & 0 & 0 \\
0 & 0 & 0 & 0
\end{array}\right), \quad A_{E_{12}}=\left(\begin{array}{cccc}
0 & 0 & 0 & 0 \\
0 & 0 & -1 & 0 \\
0 & 0 & 0 & 0 \\
0 & 0 & 0 & 0
\end{array}\right) \text {. }
\end{aligned}
$$

while the other six are of pure $B$ type (with $A=0=C$ ):

$$
\begin{gathered}
B_{E_{1}}=\left(\begin{array}{cccc}
1 & 0 & 0 & 0 \\
0 & 0 & 0 & 0 \\
0 & 0 & 0 & 0 \\
0 & 0 & 0 & -1
\end{array}\right), B_{E_{3}}=\left(\begin{array}{cccc}
0 & 0 & 0 & 0 \\
0 & -1 & 0 & 0 \\
0 & 0 & 1 & 0 \\
0 & 0 & 0 & 0
\end{array}\right), B_{E_{5}}=\left(\begin{array}{cccc}
0 & 0 & 0 & 0 \\
0 & 0 & 0 & 0 \\
-1 & 0 & 0 & 0 \\
0 & 1 & 0 & 0
\end{array}\right), \\
B_{E_{8}}=\left(\begin{array}{cccc}
0 & 0 & 0 & 0 \\
-1 & 0 & 0 & 0 \\
0 & 0 & 0 & 0 \\
0 & 0 & 1 & 0
\end{array}\right), B_{E_{10}}=\left(\begin{array}{cccc}
0 & 1 & 0 & 0 \\
0 & 0 & 0 & 0 \\
0 & 0 & 0 & -1 \\
0 & 0 & 0 & 0
\end{array}\right), B_{E_{11}}=\left(\begin{array}{cccc}
0 & 0 & 1 & 0 \\
0 & 0 & 0 & -1 \\
0 & 0 & 0 & 0 \\
0 & 0 & 0 & 0
\end{array}\right) .
\end{gathered}
$$

The correspondence with the previously introduced generators is as follows $(I=1,2,3, p=7,8)$ :

$$
P^{I} \leftrightarrow E_{I}, \quad W_{I} \leftrightarrow E_{I+3}, \quad Z_{I} \leftrightarrow E_{I+6}, \quad \Omega^{p} \leftrightarrow E_{p+3}, \quad X^{+} \leftrightarrow E_{12} .
$$

In this representation, the matrices corresponding to the negative roots,

$$
P^{-I} \leftrightarrow E_{-I}, \quad W_{-I} \leftrightarrow E_{-(I+3)}, \quad Z_{-I} \leftrightarrow E_{-(I+6)}, \quad \Omega^{-p} \leftrightarrow E_{-(p+3)}, \quad X^{-} \leftrightarrow E_{-12},
$$

are transposed with respect to the positive roots matrices:

$$
E_{-k}=\left(E_{k}\right)^{T}
$$


The following normalization conditions are assumed:

$$
\operatorname{Tr}\left(H_{i}, H_{j}\right)=4 \delta_{i j}, i, j=1 \ldots 4, \quad \operatorname{Tr}\left(E_{k}, E_{-k}\right)=2,
$$

and the involution matrix $K$ is chosen as

$$
K=\operatorname{diag}(\kappa, \kappa, 1,1,1,1, \kappa, \kappa) .
$$

The generators of the isotropy subgroup are selected by the equation $h(\Phi)^{T} K h(\Phi)=K$. They are given by the following linear combinations of the generators:

$$
P^{I}-\kappa P^{-I}, \quad Z_{I}-\kappa Z_{-I}, \quad W_{I}-W_{-I}, \quad X^{+}-\kappa X^{-}, \quad \Omega^{7}-\kappa \Omega^{-7}, \quad \Omega^{8}-\Omega^{-8} .
$$

\section{B. Matrix representation of coset $\mathscr{M}$}

$$
\mathscr{M}=\left(\begin{array}{cc}
\mathscr{P} & \mathscr{P} \mathscr{Q} \\
\mathscr{Q}^{T} \mathscr{P} & \widetilde{P}+\mathscr{Q}^{T} \mathscr{P} \mathscr{Q}
\end{array}\right),
$$

where the $4 \times 4$ blocks $\mathscr{P}$ and $\mathscr{Q}$ are

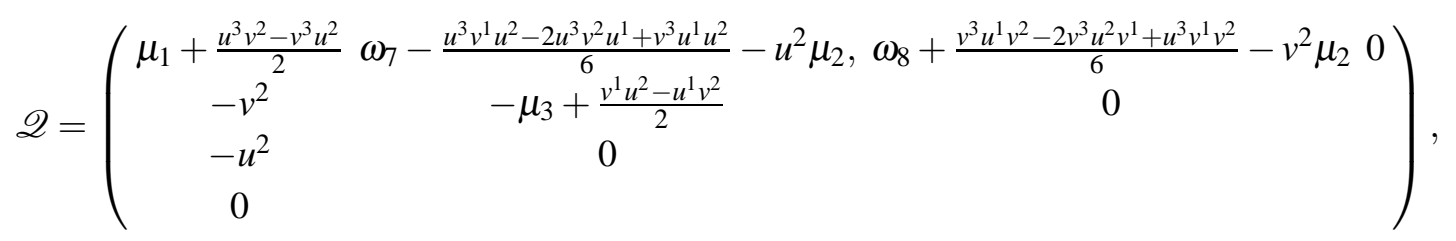

$$
\begin{aligned}
& \mathscr{P}=\left(\begin{array}{cc}
\Psi^{T} \Lambda \Psi, & \Psi^{T} \Lambda \Phi \\
\Phi^{T} \Lambda \Psi, & \Phi^{T} \Lambda \Phi+\mathrm{e}^{\sqrt{2} \phi_{4}}
\end{array}\right), \quad \widetilde{\mathscr{P}}=\left(\mathscr{P}^{-1}\right)^{\widehat{T}} .
\end{aligned}
$$

Here $\Psi$ and $\Lambda$ are the $3 \times 3$ matrices

$$
\Psi=\left(\begin{array}{ccc}
1 & u^{3} & -v^{3} \\
0 & 1 & 0 \\
0 & 0 & 1
\end{array}\right), \quad \Lambda=\kappa\left(\begin{array}{ccc}
\mathrm{e}^{\sqrt{2} \phi_{1}} & 0 & 0 \\
0 & \mathrm{e}^{\sqrt{2} \phi_{2}} & -\chi \mathrm{e}^{\sqrt{2} \phi_{2}} \\
0 & -\chi \mathrm{e}^{\sqrt{2} \phi_{2}} & \mathrm{e}^{\sqrt{2} \phi_{2}} \chi^{2}+\kappa \mathrm{e}^{\sqrt{2} \phi_{3}}
\end{array}\right)
$$

and $\Phi$ is the 3-column

$$
\Phi=\left(\begin{array}{c}
\mu_{2}+\frac{1}{2}\left(u^{1} v^{3}-u^{3} v^{1}\right) \\
-v^{1} \\
-u^{1}
\end{array}\right)
$$

\section{References}

[1] R. Emparan and H.S. Reall,Class. Quant. Grav. 23, R169 (2006) [arXiv:hep-th/0608012]; Living Rev. Rel. 11, 6 (2008) [arXiv:0801.3471].

[2] Gerard Clement, "Sigma-model approaches to exact solutions in higher-dimensional gravity and supergravity", Talk presented at the WE Heraeus Seminar on Models of Gravity in Higher Dimensions: From Theory to Experimental Search, Bremen, 25-29.8.2008 [arXiv:0811.0691]. 
[3] D.V. Gal'tsov, “Integrable systems in stringy gravity”, Phys. Rev. Lett. 74, 2863 (1995) [arXiv:hep-th/9410217].

[4] V. A. Belinsky and V. E. Zakharov, "Integration Of The Einstein Equations By The Inverse Scattering Problem Technique And The Calculation Of The Exact Soliton Solutions," Sov. Phys. JETP 48, 985 (1978) [Zh. Eksp. Teor. Fiz. 75, 1953 (1978)]. V. A. Belinsky and V. E. Zakharov, "Stationary Gravitational Solitons With Axial Symmetry," Sov. Phys. JETP 50, 1 (1979) [Zh. Eksp. Teor. Fiz. 77, 3 (1979)].

[5] T. Harmark, "Stationary and axisymmetric solutions of higher-dimensional general relativity," Phys. Rev. D 70, 124002 (2004), [arXiv:hep-th/0408141]; R. Emparan, T. Harmark, V. Niarchos, N. A. Obers and M. J. Rodriguez, "The Phase Structure of Higher-Dimensional Black Rings and Black Holes,” JHEP 0710, 110 (2007) [arXiv:0708.2181 [hep-th]];

[6] H. Elvang and P. Figueras, "Black Saturn,” JHEP 0705, 050 (2007) [arXiv:hep-th/0701035]; H. Iguchi and T. Mishima, “Black di-ring and infinite nonuniqueness," Phys. Rev. D 75, 064018 (2007) [arXiv:hep-th/0701043]; J. Evslin and C. Krishnan, “The Black Di-Ring: An Inverse Scattering Construction," arXiv:0706.1231 [hep-th]; H. Elvang and M. J. Rodriguez, "Bicycling Black Rings," arXiv:0712.2425 [hep-th]; J. Evslin and C. Krishnan, "Metastable Black Saturns,” arXiv:0804.4575 [hep-th]. J. Evslin, JHEP 0809, 004 (2008) [arXiv:0806.3389 [hep-th]]; J. Evslin and C. Krishnan, arXiv:0706.1231 [hep-th]. H. Iguchi and T. Mishima, "Solitonic generation of five-dimensional black ring solution,” Phys. Rev. D 73, 121501 (2006) [arXiv:hep-th/0604050]; S. Tomizawa, H. Iguchi and T. Mishima, "Relationship between solitonic solutions of five-dimensional Einstein equations," Phys. Rev. D 74, 104004 (2006) [arXiv:hep-th/0608169]; S. Tomizawa and M. Nozawa, "Vaccum solutions of five-dimensional Einstein equations generated by inverse scattering method. II: Production of black ring solution," Phys. Rev. D 73, 124034 (2006) [arXiv:hep-th/0604067]; T. Koikawa, "Infinite number of soliton solutions to 5-dimensional vacuum Einstein equation,” Prog. Theor. Phys. 114, 793 (2005) [arXiv:hep-th/0501248]; T. Azuma and T. Koikawa, "Infinite number of stationary soliton solutions to five-dimensional vacuum Einstein equation,” Prog. Theor. Phys. 116, 319 (2006)

[arXiv:hep-th/0512350]; T. Mishima and H. Iguchi, "New axisymmetric stationary solutions of five-dimensional vacuum Einstein equations with asymptotic flatness," Phys. Rev. D 73, 044030 (2006) [arXiv:hep-th/0504018]; S. Tomizawa, Y. Morisawa and Y. Yasui, "Vacuum solutions of five dimensional Einstein equations generated by inverse scattering method," Phys. Rev. D 73, 064009 (2006) [arXiv:hep-th/0512252];

[7] S. S. Yazadjiev, "Magnetized static black Saturn,” arXiv:0802.0784 [hep-th]; S. S. Yazadjiev, "Completely integrable sector in 5D Einstein-Maxwell gravity and derivation of the dipole black ring solutions," [arXiv:hep-th/0602116]; S. S. Yazadjiev, "Solution generating in 5D Einstein-Maxwell-dilaton gravity and derivation of dipole black ring solutions," [arXiv:hep-th/0604140]; S. S. Yazadjiev, "Black Saturn with dipole ring," [arXiv:0705.1840 [hep-th]].

[8] A. Bouchareb, G. Clément, C-M. Chen, D. V. Gal'tsov, N. G. Scherbluk, and Th. Wolf, “G2 generating technique for minimal 5D supergravity and black rings”, Phys. Rev. D 76, 104032 (2007) [arXiv:0708.2361].

[9] G. Clément, Journ. Math. Phys. 49, 042503 (2008); Erratum, Journ. Math. Phys. 49, 079901 (2008) [arXiv:0710.1192].

[10] S. Mizoguchi and N. Ohta, "More on the similarity between D = 5 simple supergravity and M theory," Phys. Lett. B 441, 123 (1998) [arXiv:hep-th/9807111]. E. Cremmer, B. Julia, H. Lü and C. N. Pope, "Higher-dimensional origin of D = 3 coset symmetries," arXiv:hep-th/9909099. S. Mizoguchi and G. Schröder, “On discrete U-duality in M-theory,” Class. Quant. Grav. 17, 835 (2000) 
[arXiv:hep-th/9909150]. M. Possel, “Hidden symmetries in five-dimensional supergravity,” PhD Thesis, Hamburg, 2003. M. Possel and S. Silva, "Hidden symmetries in minimal five-dimensional supergravity," Phys. Lett. B 580, 273 (2004) [arXiv:hep-th/0310256]. M. Gunaydin, A. Neitzke, O. Pavlyk and B. Pioline, "Quasi-conformal actions, quaternionic discrete series and twistors: $S U(2,1)$ and $G_{2}(2)$ " [arXiv:0707.1669]

[11] M. Gunaydin and F. Gursey, “Quark structure and octonions,” J. Math. Phys. 14, 1651 (1973).

[12] D.V. Gal'tsov and N.G. Scherbluk, “Generating technique for $U(1)^{3} 5 D$ supergravity," Phys. Rev. D 78, 064033 (2008) [arXiv:0805.3924].

[13] A. A. Pomeransky and R. A. Sen'kov, "Black ring with two angular momenta," arXiv:hep-th/0612005.

[14] H. Ishihara and K. Matsuno, “ Kaluza-Klein black holes with squashed horizons,” Prog. Theor. Phys. 116 417, (2006) [arXiv: hep-th/0510094].

[15] R. G. Cai, L. M. Cao and N. Ohta, Phys. Lett. B 639, 354 (2006) [arXiv:hep-th/0603197].

[16] Y. Kurita and H. Ishihara, "Mass and Free energy in Thermodynamics of Squashed Kaluza-Klein Black Holes," Class. Quant. Grav. 24, 4525 (2007) [arXiv:0705.0307 [hep-th]]; "Thermodynamics of Squashed Kaluza-Klein Black Holes and Black Strings - A Comparison of Reference Backgrounds," Class. Quant. Grav. 25, 085006 (2008) [arXiv:0801.2842 [hep-th]].

[17] T. Wang, "A Rotating Kaluza-Klein black hole with squashed horizons," Nucl. Phys. B 756 86-99, (2006) [arXiv: hep-th/0605048].

[18] T. Nakagawa, H. Ishihara, K. Matsuno, and S. Tomizawa, "Charged Rotating Kaluza-Klein Black Holes in Five Dimensions,” Phys. Rev. D 77,044040, (2008) [arXiv:0801.0164].

[19] S. Tomizawa, H. Ishihara, K. Matsuno, and T. Nakagawa, "Squashed Kerr-Godel Black Holes: Kaluza-Klein Black Holes with Rotations of Black Hole and Universe,” [arXiv:0803.3873]. (2008)

[20] K. Matsuno, H. Ishihara, T. Nakagawa, and S. Tomizawa, "Rotating Kaluza-Klein Multi-Black Holes with Godel Parameter,” Phys. Rev. D 78,064016, (2008) [arXiv:0806.3316].

[21] S. Tomizawa and A. Ishibashi, "Charged Black Holes in a Rotating Gross-Perry-Sorkin Monopole Background,” [arXiv:0807.1564]. (2008)

[22] S. Tomizawa, Y. Yasui, and Y. Morisawa, "Charged Rotating Kaluza-Klein Black Holes Generated by G2(2) Transformation,” [arXiv:0809.2001]. (2008)

[23] D. Rasheed, “The rotating dyonic black holes of Kaluza-Klein theory," Nucl. Phys. B 454 379-401, (1995) [arXiv:hep-th/9505038].

[24] P. Fre "Lectures on Special Kähler Geometry and ElectricÜMagnetic Duality Rotations," Nucl. Phys. B (Proc. Suppl.) 45B,C (1996) 59-114 [arXiv:hep-th/9512043]

[25] P. Breitenlohner, D. Maison, and G. Gibbons, “4-Dimensional Black Holes from Kaluza-Klein theories,” Commun. Math. Phys. 120295 Ű334 (1987)

[26] P. Breitenlohner, and D. Maison, "On nonlinear sigma models arising in (super-)gravity," Commun.Math.Phys. 209:785-810, (2000) [arXiv:gr-qc/9806002]

[27] L. Andrianopoli, M. Bertolini, Anna Ceresole, R. D’Auria, S. Ferrara, P. Fre, T. Magri “N=2 supergravity and $\mathrm{N}=2$ superYang-Mills theory on general scalar manifolds: Symplectic covariance, gaugings and the momentum map," J. Geom. Phys. 23 111-189, (1997) [arXiv:hep-th/9605032] 